\title{
Educação continuada: um levantamento de necessidades da equipe de enfermagem*
}

Continuing education: a survey of needs for the nursing team

Milena Froes da Silva', Fabiana Alves da Conceição², Maria Madalena Januário Leite

\section{Resumo}

Este estudo teve como objetivo fazer um diagnóstico sobre o Serviço de Educação Continuada (SEC) de um hospital público de São Paulo, a fim de identificar necessidades das equipes de enfermagem que atuam nas diferentes Unidades de Terapia Intensiva (UTIs). Esse serviço, subordinado à diretoria do hospital, realiza atividades educativas com periodicidade variável. Com um levantamento das necessidades sentidas por membros dessas equipes de enfermagem, através de opiniões sobre o modo como o SEC tem operado, seria possível identificar fatores favoráveis, ou desfavoráveis, para a inserção de enfermeiros das UTIs nas ações educativas para o treinamento prático in loco de outros trabalhadores. Espera-se que isso possa contribuir para melhorar o respectivo desempenho e desenvolvimento profissional e também para melhorar a autoestima dos enfermeiros assistenciais exercendo atividades educacionais, não só na própria unidade, mas eventualmente em outras promovidas pelo SEC.

Palavras-chave: Educação continuada; equipe de enfermagem; desenvolvimento de pessoal.

\begin{abstract}
This study had the objective to diagnose the Continuing Education Service (CES) of a public hospital in São Paulo, in order to identify needs of the nursing teams working at different Intensive Care Unit (ICU). This service, subordinated to the hospital director, carries out educational activities in a variable periodicity. With a survey on nursing teams, members felt needs through opinions on ways the CES has operated, it would be possible identifying favorable or unfavorable factors for insertion of the ICU nurses into educational actions for practical training of workers on site. Analyzing the survey of needs noticed by nursing team members, through theirs opinions on ways the CES has operated, it would be possible identifying favorable or unfavorable factors for insertion of ICU nurses into educational actions for practical on site training of workers. It is expected that this will contribute for improvement of professional performance and development and also to improve the nurses self-esteem while exerting educational activities at ICU, not only at their proper unities, but also eventually in others promoted by CES.
\end{abstract}

Keywords: Education, continuing; nursing, team; staff development.

Recebido: $21 / 2 / 2008$

Revisado: $11 / 11 / 2008$

Aprovado: 22/1/2009

\footnotetext{
* Parte de monografia de conclusão do Segundo Curso de Especialização em Gerenciamento de Serviços de Saúde da Escola de Enfermagem da Universidade de São Paulo (USP), São Paulo (SP)

Departamento de Orientação Profissional da Escola de Enfermagem da Universidade de São Paulo (USP), São Paulo (SP)

2 Unidade de Terapia Intensiva do Hospital Estadual Mário Covas, Santo André (SP)

Endereço para correspondência: Milena Froes da Silva - Avenida Dr. Enéas de Carvalho Aguiar, 419 - Cerqueira César - CEP: $05403-000$ - São Paulo

(SP) - Fone: (11) 3061-7574 - E-mail: milenafroes@usp.br
} 


\section{Introdução}

A educação continuada é componente essencial dos programas de formação e desenvolvimento de recursos humanos das instituições. Oguisso $^{1}$ afirma que, sendo o capital humano o elemento mais importante no funcionamento de qualquer empresa, grande ou pequena, pública ou privada, o mesmo deve ser objeto de análises permanentes e de adequação de funções para melhorar a eficiência do trabalho, a competência profissional e o nível de satisfação do pessoal.

De acordo com a Organização Panamericana de Saúde, educação continuada é um processo dinâmico de ensino-aprendizagem, ativo e permanente, destinado a atualizar e melhorar a capacitação de pessoas ou grupos, face à evolução científico-tecnológica, às necessidades sociais e aos objetivos e metas institucionais. Assim, a educação continuada precisa ser considerada como parte de uma política global de qualificação dos trabalhadores, centrada nas necessidades de transformação da prática ${ }^{1}$.

A Organização Mundial de Saúde ${ }^{2}$ reconhece a educação continuada como essencial para a qualidade da assistência à saúde. Segundo Oguisso $^{1}$, a OMS entende que a educação continuada auxilia no desenvolvimento dos recursos humanos, num esforço sistemático de melhorar o funcionamento dos serviços através do desempenho do seu pessoal.

Atualmente, o Ministério da Saúde aponta alguns programas utilizados pelos Serviços de Educação Continuada (SEC) das instituições de saúde que possuem limitada capacidade de produzir impacto sobre as instituições formadoras, no sentido de alimentar os processos de mudança, já que mantém a lógica programática das ações, não desafiando os distintos atores para uma postura de mudança e problematização de suas próprias práticas e do trabalho em equipe. Assim, para minimizar essas deficiências, propõe a implementação da educação permanente em saúde, que tem por desafio constituir-se em: eixo transformador; estratégia mobilizadora de recursos e poderes; recurso estruturante do fortalecimento dos serviços de educação das instituições de saúde 3 .

A educação permanente em saúde é uma nova estratégia para a formação e desenvolvimento das práticas educativas, devendo ser tomada como um recurso inovador para a gestão do trabalho, entendendo que o aprender e ensinar se incorporam ao quotidiano das organizações e ao trabalho. Nessa proposta, a mudança das estratégias de organização e do exercício da atenção terá que ser construída na prática concreta das equipes ${ }^{3}$.

As demandas para capacitação não se definem somente a partir de uma lista de necessidades individuais de atualização, nem das orientações dos níveis centrais, mas prioritariamente a partir dos problemas que acontecem no dia-a-dia do trabalho referentes à atenção à saúde e organização do trabalho, considerando a necessidade de prestar ações e serviços relevantes e de qualidade. É a partir da problematização do processo e da qualidade do trabalho - em cada serviço de saúde - que são identificadas as necessidades de capacitação, garantindo a aplicabilidade e a relevância dos conteúdos e tecnologias estabelecidas ${ }^{3}$.

O marco conceitual da educação permanente é o de aceitar que formação e desenvolvimento devem ser feitos de modo: descentralizado e transdisciplinar, que propicie a democratização institucional, o desenvolvimento da capacidade de aprendizagem, a melhora permanente da qualidade do cuidado à saúde e a constituição de práticas técnicas críticas, éticas e humanísticas. Desse modo, transformar a formação e gestão da educação em saúde não pode ser considerada questão simplesmente técnica, já que envolve mudanças nas relações, nos processos, nos atos de saúde e, principalmente, nas pessoas.

Nesse contexto, a educação continuada pode configurar-se como um campo de captação e propagação de conhecimentos, práticas e reflexões sobre o processo de trabalho da enfermeira e da equipe de enfermagem.

Dessa forma, evidencia-se o importante papel da educação continuada na mobilização das potencialidades dos trabalhadores de enfermagem, pois ao resgatar uma concepção voltada para o desenvolvimento desses profissionais, permite uma melhor compreensão da experiência, da identidade e dos saberes dos mesmos ${ }^{4}$.

O desenvolvimento de um processo educativo junto a adultos no seu ambiente de trabalho ou para o seu trabalho, deve levar em consideração que o ponto de partida da aprendizagem é a experiência adquirida por esses profissionais em sua vida diária e que cada um já vem com uma bagagem específica. Ao assumir sua profissão, o trabalhador precisa integrar "seu jeito de ser", adaptando suas características pessoais à cultura organizacional. Para isso o profissional deve estar engajado e querer crescer, pois o aprendizado se dá pela conscientização ${ }^{5}$. Como afirma Freire ${ }^{6}$ " “A conscientização não é apenas conhecimento ou reconhecimento, mas opção, decisão e compromisso".

Também diante das novas exigências das organizações de saúde, a enfermagem enfrenta contínuas transformações, o que implica que as pessoas precisam procurar a melhor forma de ampliar seus conhecimentos, e um dos caminhos é a educação e a aprendizagem contínua ${ }^{4,7}$. O papel do processo educativo na enfermagem é a formação de trabalhadores com uma visão mais crítica e reflexiva de suas ações, a fim de que os mesmos possam construir sua realidade, articulando teoria e prática.

Kurcgant $^{8}$ alerta para a tendência dos serviços de enfermagem de buscarem modelos de programas educativos que, muitas vezes, são dissociados da realidade e que nem sempre alcançam os resultados esperados. Na nossa prática, enquanto enfermeiras, concordamos com a afirmação de Kurcgant, pois atualmente os programas de aperfeiçoamento visam assegurar a eficiência e a atualização do funcionário, desenvolvendo-o para atividades específicas.

Dalvim, Torres e Santos verificaram em sua prática como docentes assistenciais em uma instituição pública de saúde, certo distanciamento dos enfermeiros em relação às ações educativas, bem como uma 
restrita visão no que se refere aos problemas e necessidades educacionais da equipe de enfermagem como um todo.

Cadete e Villa ${ }^{10}$ observaram que alguns enfermeiros têm buscado um fazer diferente, um fazer que leve o outro a pensar e procurar, por si mesmo, visualizar o que pode ser mudado. As ações de enfermagem indicam haver um trabalho educativo dentro de uma nova abordagem, baseadas numa aprendizagem inovadora, que leve o educando a refletir nas suas próprias possibilidades. Para as autoras, a condução do processo ensino-aprendizagem sob essa ótica, demanda tempo e experiência para que se possa consolidar a proposta. Contudo, apesar da nova visão de "como educar", os enfermeiros expressam a dificuldade em trabalhar de maneira diferente e reiteram a velha forma no fazer diário.

Ainda existe um grande campo a ser conquistado pelos serviços de educação continuada, porém o estímulo para essa conquista também deve vir de nós mesmos, enfermeiros, que compõem a equipe de trabalho. A busca contínua por conhecimento, criatividade e pela capacidade de tomar as decisões certas são algumas das atitudes que esperamos encontrar nos enfermeiros que compõem esse serviço.

Garrido $^{11}$ ressalta a necessidade de se reiterar as possibilidades de criar, aprender, se renovar, num trabalho educativo no qual o educador e o educando têm muito a contribuir no processo ensino-aprendizagem. Devemos considerar a equipe de enfermagem como sujeitos da sua própria educação, não esquecendo que os enfermeiros do serviço de educação continuada devem ser capacitados para desempenhar o papel de educador, tendo a consciência da realidade na qual estão inseridos, pois ninguém pode estar no mundo, com o mundo e com os outros de forma neutra ${ }^{12}$.

As ações dos programas de educação continuada devem estimular nos educandos o repensar sobre os valores profissionais, que resulte na melhoria do processo de cuidar. Garrido ${ }^{11}$ afirma que a educação continuada deve surgir como fator determinante para a mudança, permeando a ciência do ser enfermeiro, proporcionando a aquisição de habilidades, autoconfiança, crescimento profissional e incentivo. É a educação que leva ao cuidar e o cuidar que leva à educação.

O desenvolvimento das pessoas é um dos fatores que pode assegurar a qualidade do atendimento ao cliente e a sobrevivência da instituição neste cenário de mudanças e competitividade. Para Boff $\mathrm{f}^{13}$, "No processo de nossa vida, lentamente vamos conquistando nosso ser, nosso lugar na sociedade, nossa profissão, nossos objetivos de curto e longo prazo. É uma árdua caminhada”.

Diante do exposto, verifica-se que é de fundamental importância o constante aprimoramento dos profissionais de saúde, ressaltando-se o papel do SEC dentro das instituições, bem como o entendimento que as equipes de trabalho tem a respeito desse serviço.

Dessa forma, considerando as fases do planejamento do SEC que, segundo Gonçalves ${ }^{14}$ compreende: diagnóstico, objetivos/metodologia, avaliação/implementação e análise dos recursos/custos. Iniciamos a investigação a partir da fase de diagnóstico em que é feito o levantamento de necessidades da equipe de enfermagem das
Unidades de Terapia Intensiva (UTIs) de um hospital público da grande São Paulo, quanto ao desenvolvimento de um programa de educação continuada na instituição, bem como os fatores que favorecem e os que dificultam a inserção dos enfermeiros assistenciais das UTIs, nas ações educativas in locu e naquelas que são promovidas pelo SEC do hospital.

As atividades de educação continuada na UTI, mesmo restringindo-se às necessidades do enfermeiro, são deficientes dada à presença da alta tecnologia e o constante desafio de aprendê-la prontamente, sendo este um desafio tanto para os enfermeiros das unidades quanto para os enfermeiros de educação em serviço ${ }^{15}$.

Os objetivos deste estudo foram: verificar a percepção da equipe de enfermagem em relação ao SEC da instituição; identificar as necessidades da equipe de enfermagem das UTIs do Hospital Estadual Mário Covas, quanto ao desenvolvimento de um programa de educação continuada na instituição; verificar a opinião dos enfermeiros e técnicos de enfermagem quanto à contribuição dos programas de educação continuada para o desenvolvimento profissional dentro da instituição e identificar os fatores que favorecem e os que dificultam a inserção dos enfermeiros assistenciais das UTIs nas ações educativas in locu e naquelas que são promovidas pelo SEC da instituição.

\section{Casuística e métodos}

O estudo realizado foi do tipo descritivo e exploratório, pois apresentou e identificou sistematicamente as características do fenômeno em questão, ou seja, as impressões dos sujeitos de pesquisa sobre o SEC da instituição em estudo, e exploratório por buscar maiores informações sobre $o$ assunto ${ }^{16}$.

\section{Local}

A pesquisa foi desenvolvida no Hospital Estadual Mário Covas, localizado no município de Santo André na Grande São Paulo. A instituição tem como filosofia a assistência, o ensino e a pesquisa com atenção terciária, exclusiva aos pacientes do Sistema Único de Saúde (SUS), realizando procedimentos diagnósticos e terapêuticos de alta complexidade à população do grande $\mathrm{ABC}$ (Santo André, São Bernardo do Campo, São Caetano do Sul, Diadema, Mauá, Ribeirão Pires e Rio Grande da Serra).

O hospital é considerado uma Organização Social de Saúde (OSS), com administração descentralizada, de médio porte, gerenciado pela Fundação do ABC que é a mantenedora de Faculdade de Medicina ABC. Conta com 261 leitos e possui quatro UTIs. Nas UTIs a equipe de enfermagem é composta, exclusivamente, por 60 técnicos de enfermagem e 15 enfermeiros, que desenvolvem diferentes técnica complexas.

O SEC da instituição é um serviço de assessoria (ou staff) responsável pelo treinamento, capacitação e reciclagem dos profissionais de enfermagem de toda a instituição. 


\section{Sujeitos da pesquisa}

A população do estudo foi constituída por 75 pessoas, sendo 15 enfermeiros e 60 técnicos de enfermagem e a amostra foi de 68 pessoas, sendo 11 enfermeiros e 57 técnicos de enfermagem dos quatro turnos de trabalho (manhã, tarde e noturno par e ímpar), que integram as quatro UTIs do hospital (geral, coronariana e pediátrica) e que atenderam aos seguintes critérios de inclusão: possuir mais de um ano de exercício profissional na instituição; e não estar de férias ou licença durante o período de coleta de dados.

\section{Coleta de dados}

O instrumento de coleta de dados foi composto por um questionário (Anexo I) com duas partes. A primeira parte do instrumento foi igual para ambas categorias profissionais (enfermeiros e técnicos de enfermagem), sendo composta pela caracterização dos indivíduos e pelas questões de múltipla escolha relacionadas ao SEC; já a segunda parte foi aplicada somente para os enfermeiros, por ser tratar de perguntas abertas relacionadas aos fatores que favorecem e os que dificultam a atuação dos mesmos no processo educativo da equipe.

Os questionários foram distribuídos pelas pesquisadoras, na primeira semana do mês de junho de 2007, sendo recolhidos 20 dias após a entrega.

\section{Tratamento e análise dos dados}

A apresentação dos resultados se fez de duas formas: em relação aos questionários foram analisadas quantitativamente as respostas contidas nos mesmos, avaliando a existência de dependência entre as variáveis quantitativas do estudo; no tratamento estatístico adotado realizou-se a análise exploratória por meio de frequências absolutas e relativas (\%) para exploração e conhecimento da amostra: idade, sexo, formação, função, tempo de formado, tempo na instituição, tempo de experiência em UTI e turno de trabalho e tabelas de contingência para cruzamento das variáveis de interesse.

Com relação à pergunta aberta que foi aplicada aos enfermeiros, fizemos o levantamento quantitativo das respostas obtidas e posteriormente a discussão de todo material.

\section{Aspectos éticos}

O projeto de pesquisa foi analisado e aprovado pelos pelas Comissões de Ética em Pesquisa do Hospital Mário Covas e da Faculdade de Medicina ABC (Parecer 081/2007). Em seguida os integrantes da equipe de enfermagem das UTIs do hospital foram abordados individualmente, durante o mês de junho e, após esclarecimento dos seus objetivos, os que concordaram participar assinaram o termo de consentimento livre e esclarecido, conforme as normas da resolução 196/96 sobre as diretrizes e normas regulamentares de pesquisa em seres humanos ${ }^{17}$.

Foi assegurado o esclarecimento de todas as dúvidas que poderiam existir sobre a pesquisa e que o participante poderia retirar sua participação a qualquer momento, sem qualquer prejuízo, e que os dados que poderiam revelar-lhe individualmente não seriam divulgados em nenhum relatório ou publicação.

\section{Resultados e discussão}

Foram entregues 75 questionários e tivemos a devolutiva de apenas 68 com uma recusa. Inicialmente foi feita a caracterização da população do estudo, sendo que quanto à idade, foi verificado que a maioria (48,5\%) possui de 21 a 30 anos e apenas $2,9 \%$ possuem mais de 50 anos. Em relação ao sexo, 67,6\% dos profissionais são mulheres e 32,4\% são homens. Quanto à formação, $57,3 \%$ possuem somente o ensino médio. Na variável função, $85,3 \%$ são técnicos de enfermagem e 14,7\% são enfermeiros. Quanto ao tempo de formado, a maioria dos profissionais (52,9\%) possui entre dois a cinco anos de formado. Nas variáveis tempo na instituição* e tempo de experiência em UTI, verificou-se que a maioria dos participantes do estudo, 55,6\% e 57,3, respectivamente, compõem a parcela de maior representatividade para o período de tempo estabelecido, que foi de um a três anos. O turno de trabalho foi o último item abordado na caracterização e nele foi observado que $29,4 \%$ dos profissionais trabalham no período matutino, $27,9 \%$ no vespertino e $42,7 \%$ no período da noturno.

Ao relacionarmos a variável função com o tempo de experiência em UTI, obtivemos os dados apresentados na Tabela 1. Como foi possível observar, $41,4 \%$ dos técnicos de enfermagem possuem até um ano de experiência em UTI e $50 \%$ dos enfermeiros possuem de três anos e meio à aproximadamente cinco anos de experiência em UTI, o que caracteriza a UTI do hospital como um setor composto, em sua maioria,

Tabela 1 - Distribuição da variável função de acordo com o tempo de experiência em UTI (São Paulo, 2007)

\begin{tabular}{|c|c|c|c|c|c|}
\hline \multirow[b]{2}{*}{ Função } & \multicolumn{5}{|c|}{ Tempo de experiência em UTI } \\
\hline & $\begin{array}{c}\leq 1 \text { ano } \\
(\%)\end{array}$ & $\begin{array}{c}13 \text { meses a } 3 \text { anos } \\
\text { e } 5 \text { meses } \\
(\%)\end{array}$ & $\begin{array}{c}3 \text { anos e } 6 \text { meses } \\
\text { a } 5 \text { anos e } 5 \text { meses } \\
(\%)\end{array}$ & $\begin{array}{c}5 \text { anos e } 6 \text { meses } \\
\text { a } 10 \text { anos } \\
(\%)\end{array}$ & $\begin{array}{c}10 \text { anos e } 1 \text { mês } \\
\text { a } 24 \text { anos } \\
(\%)\end{array}$ \\
\hline Técnicos de Enfermagem & 41,4 & 19 & 24,1 & 8,6 & 6,9 \\
\hline Enfermeiros & 20 & 20 & 50 & 0 & 10 \\
\hline
\end{tabular}

\footnotetext{
* Respeitando o critério de exclusão, somente os funcionários com mais de um ano de atuação na instituição participaram do estudo, assim 29,2\% da amostra não foi analisada.
} 
por técnicos de enfermagem com pouca experiência no cuidado de enfermagem a pacientes críticos, mas com enfermeiros que detêm maior tempo de experiência nessa especialidade.

Após a caracterização da população e respondendo ao primeiro objetivo, que foi identificar a percepção da equipe de enfermagem em relação ao SEC da instituição, foram feitas análises quantitativas e cruzamentos dos dados obtidos nos questionários, para o qual o instrumento propôs ao indivíduo questões relacionadas ao seu conhecimento sobre educação continuada e suas funções, bem como seu desenvolvimento profissional. Na primeira questão "Quando foi a última vez que você participou de um curso ou evento de aprimoramento?", 91,2\% responderam que participam de cursos e eventos. Ao relacionar com a variável participação em curso ou eventos com a função, obtivemos a Tabela 2.

Conforme a Tabela 2, 93,1\% dos técnicos de enfermagem e 80\% dos enfermeiros afirmaram participar de cursos ou eventos. Ainda sobre a questão participação em cursos ou eventos, foi questionado quando foi a última vez que os entrevistados participaram de um curso ou evento de aprimoramento e 77,9\% responderam que foi há menos de três meses, $5,9 \%$ entre três a seis meses, $5,9 \%$ entre seis meses a um ano e 8,8\% há mais de um ano. Correlacionando este dado com a variável função, percebeu-se que $84,2 \%$ daqueles que afirmaram a participação em cursos ou eventos há menos de três meses são técnicos de enfermagem e apenas $15,8 \%$ são enfermeiros.

Nas questões relacionadas ao SEC e suas funções, apreende-se que os entrevistados, em quase sua totalidade ( $96 \%)$, afirmam saber o que é SEC. Ainda neste item, foi pedido que os mesmos assinalassem a(s) alternativa(s) que melhor expressassem a função do SEC na instituição do estudo. Sobre isto, $55,9 \%$ assinalaram o item auxiliar o profissional a adquirir novos conhecimentos, $50 \%$ o item treinar a equipe de enfermagem sobre determinado assunto, $35,3 \%$ assinalou o item atuar no processo de recrutamento de profissionais, e o item criar condições que favoreçam as modificações e atitudes e 1,5\% referem que o SEC atua na integração dos profissionais na instituição. Do total de técnicos de enfermagem, somente $16,2 \%$ assinalaram todos os itens e do total de enfermeiros somente $18,2 \%$ assinalaram todos os itens, o que supõe que poucos profissionais entendem qual é a real função do SEC na instituição.
Ao cruzarmos a questão "você considera que o SEC da instituição é totalmente responsável pelo seu desenvolvimento profissional?” com a variável função, observamos que $70,7 \%$ dos técnicos de enfermagem e $90 \%$ dos enfermeiros não consideram o SEC totalmente responsável pelo seu desenvolvimento profissional.

Quanto ao segundo objetivo, que foi identificar as necessidades da equipe de enfermagem das UTIs do hospital quanto ao desenvolvimento de um programa de educação continuada na instituição, foram feitas análises quantitativas e cruzamento dos dados obtidos no questionário. Relacionando a variável função com e questão "assinale a(s) alternativa(s) que melhor define (m) o que a instituição pode fazer para contribuir como o seu desenvolvimento profissional”, observamos que $56 \%$ dos técnicos de enfermagem e $50 \%$ dos enfermeiros não consideram que a instituição deva financiar cursos de aprimoramento fora da instituição; $82,8 \%$ dos técnicos de enfermagem e $60 \%$ dos enfermeiros consideram que a instituição deve promover cursos de aprimoramento dentro da instituição.

Em relação a acrescentar carga horária dos cursos realizados em banco de horas, $81 \%$ dos técnicos de enfermagem e 70\% dos enfermeiros não consideram importante. Contudo, do total de $49 \%$ dos entrevistados que consideram importante este item, $64,2 \%$ pertencem ao turno de trabalho noturno.

No que diz respeito à participação em cursos como um critério positivo na avaliação dos planos de carreira, $51,7 \%$ dos técnicos de enfermagem consideram importante, diferentemente de $80 \%$ dos enfermeiros.

Cruzando a variável função com a questão "Que (quais) programa(s) de capacitação promovido (s) pelo SEC desta instituição atenderia as suas necessidades?, obtivemos a Tabela 3, na qual observase que, praticamente, $60 \%$ dos profissionais não consideram necessário/importante a promoção de cursos que abordem questões éticas e

Tabela 2 - Distribuição da variável participação em cursos ou eventos de acordo com a função (São Paulo, 2007)

\begin{tabular}{lcc}
\hline \multirow{2}{*}{ Função } & \multicolumn{2}{c}{ Participação em cursos ou eventos } \\
\cline { 2 - 3 } & Sim (\%) & Não (\%) \\
\hline Técnicos de Enfermagem & 93,1 & 6,9 \\
Enfermeiros & 80 & 20 \\
\hline
\end{tabular}

Tabela 3 - Distribuição da variável função com a resposta à pergunta "que (quais) programa(s) de capacitação promovido(s) pelo Serviço de Educação Continuada desta instituição atenderia às suas necessidades?" (São Paulo, 2007)

\begin{tabular}{|c|c|c|c|c|}
\hline \multirow[b]{2}{*}{ Programas de capacitação } & \multicolumn{2}{|c|}{ Enfermeiros } & \multicolumn{2}{|c|}{ Técnicos de enfermagem } \\
\hline & $\begin{array}{l}\text { Sim } \\
(\%)\end{array}$ & $\begin{array}{l}\text { Não } \\
\text { (\%) }\end{array}$ & $\begin{array}{l}\text { Sim } \\
(\%)\end{array}$ & $\begin{array}{l}\text { Nãc } \\
(\%)\end{array}$ \\
\hline Liderança & 50 & 50 & 27,6 & 72,4 \\
\hline Ética & 40 & 60 & 37,9 & 62,1 \\
\hline Comunicação & 40 & 60 & 34,5 & 65,5 \\
\hline Sistematização da assistência de enfermagem & 30 & 70 & 43,1 & $56, \mathrm{c}$ \\
\hline Legislação & 50 & 50 & 24,1 & 75,9 \\
\hline Relacionamento interpessoal & 30 & 70 & 50 & 50 \\
\hline Relacionamento com paciente e acompanhante & 30 & 70 & 36,2 & 63,8 \\
\hline Técnicas específicas para UTI & 40 & 60 & 81 & 19 \\
\hline Ações voltadas à prevenção e controle de infecções hospitalares & 30 & 70 & 58,6 & 41,4 \\
\hline
\end{tabular}


de comunicação; relacionamento interpessoal e relacionamento com o paciente e acompanhante.

Sobre essas questões, um estudo realizado por Santos e Silva ${ }^{18}$ sobre a percepção dos profissionais de saúde sobre a comunicação com familiares de pacientes em UTIs, demonstrou que os profissionais de enfermagem de nível médio sentem que a família não valoriza suas informações, porque para a família o mais importante é conversar com o médico. Outros fatores abordados no estudo que dificultam a relacionamento com o paciente e acompanhante é a própria dinâmica da UTI, o grau de desconhecimento pelo profissional acerca da evolução clínica do paciente, além do desconhecimento do modo de ser e perceber da família e as dificuldades inerentes ao próprio jeito de ser do profissional.

A autora ainda ressalta a importância da existência de suporte e treinamento para os profissionais conseguirem desenvolver uma comunicação mais efetiva com os familiares e poderem atender a família como um núcleo que também precisa de cuidados.

Ao compararmos as porcentagens obtidas no item sistematização da assistência de enfermagem, verificou-se que 70\% dos enfermeiros não a consideram importante, por outro lado $43,1 \%$ dos técnicos de enfermagem consideram importante essa temática para cursos de capacitação, o que nos chama a atenção, visto que na prática profissional esse tema tem gerado muitas discussões e estudos quanto ao entendimento de sua efetiva aplicabilidade.

Ainda respondendo sobre as necessidades da equipe quanto ao SEC, ressalta-se a fala de um colaborador técnico de enfermagem, que enfatizou a extrema necessidade que a equipe tem em aprimorar seus conhecimentos, através da realização de discussões de casos semanais, pois “... infelizmente, ao longo dos dias, essa atividade vai ficando cada vez mais impossível; os profissionais estão sobrecarregados, com um número excessivo de clientes e o serviço acaba ficando robotizado e mecânico...”.

Quanto ao terceiro objetivo do trabalho, que foi identificar os fatores que favorecem e os que dificultam a inserção dos enfermeiros assistenciais das UTIs nas ações educativas in locu e naquelas que são promovidas pelo SEC da instituição, de 15 questionários entregues, 67\% foram devolvidos com a parte II respondida. A pergunta "Quais são os fatores que favorecem e os que dificultam a sua inserção nas ações educativas in locu?"

A partir das respostas, foram classificadas as dificuldades e facilidades em fatores estruturais e processo de trabalho. Nomeamos como fatores estruturais as respostas que fizeram referência às oportunidades oferecidas pela instituição, bem como a inexistência destas; e como processo de trabalho que traduziram a percepção dos indivíduos sobre a sua atuação na instituição, ressaltando seus pontos positivos e negativos.

Quanto aos fatores que favorecem, foram classificadas como estruturais a realização de atividades durante o período de trabalho e campo rico em materiais e diagnósticos; e como processo de trabalho a proximidade e o contato diário entre o enfermeiro e o técnico, interesse pessoal e da equipe.
Quanto aos fatores que dificultam, foram classificadas como estruturais a sobrecarga de atividades voltadas à assistência; o número insuficiente de funcionários e número excedente de pacientes; os problemas administrativos e burocráticos que afastam o enfermeiro das atividades educativas; treinamento fora do horário de trabalho; não disponibiliza banco de horas; temas impostos pela instituição e ausência de subsídios para promover ações educativas; e como processo de trabalho a falta de planejamento e administração do tempo, sendo que a educação continuada não estimula a promoção de ações educativas.

Mediante esta classificação, observa-se que os fatores estruturais dificultam a adesão/inserção dos enfermeiros nas ações educativas in loco, e o processo de trabalho foi o mais citado como facilitador para as ações educativas do enfermeiro, visto que este pode atuar diretamente sobre as necessidades do trabalhador no momento em que este executa suas atividades, percebendo o real interesse da equipe diante das situações cotidianas.

Para a pergunta "Quais são os fatores que favorecem e os que dificultam a sua inserção nas ações educativas junto ao SEC do hospital?”, também utilizou-se a mesma classificação, fatores estruturais e processo de trabalho, e observamos que a dificuldade do enfermeiro em atuar junto ao SEC deve-se aos fatores estruturais relativos ao horário de atuação do Serviço, excesso de atividades da enfermeira responsável por este serviço, ausência de parceria entre enfermeiros assistenciais e enfermeira da educação continuada, o que acarreta em desinteresse do grupo em participar das atividades de capacitação desenvolvidas, pois, segundo as falas dos entrevistados, as mesmas pouco contribuem para o aprimoramento da equipe, visto que são repetitivas e atendem, prioritariamente, aos interesses da instituição. E o processo de trabalho facilita devido à proximidade e o contato diário entre o enfermeiro e o técnico, que permite o diagnóstico das necessidades da equipe, indivíduo e a intervenção imediata, propiciando a capacitação da equipe através de atualizações constantes promovidas pelo SEC com consequente melhora da assistência prestada, estimulando o interesse pessoal e da equipe em participar das atividades educativas.

Visto a importância do SEC nas instituições de saúde, observa-se que muitos profissionais, inclusive o enfermeiro, desconhecem a finalidade do mesmo, e esse fato foi evidenciado na primeira questão na qual somente $16,2 \%$ assinalaram todos os itens, sendo que destes $81,8 \%$ são técnicos de enfermagem e 18,2\% enfermeiros, o que supõe que poucos profissionais entendem qual é a real função do SEC na instituição. Acredita-se que esse "desconhecimento" é resultado das poucas discussões acerca desse tema durante a graduação, como também da não informação nos cursos de nível médio; em consequência a esse fato, é observado que alguns profissionais acreditam ser de total responsabilidade desse Serviço o seu desenvolvimento profissional, ou seja, 29,3\% dos técnicos de enfermagem e $10 \%$ dos enfermeiros transferiram toda a responsabilidade para a instituição. No entanto, acredita-se que o treinamento não pode ser apenas um meio para o funcionário capacitar-se para o trabalho, deve ser um instrumento que auxilie o profissional a refletir sobre a importância do 
seu trabalho e quanto ele pode ser rico no seu dia-a-dia, devendo sempre motivá-lo à busca de enriquecimento profissional.

Assim, a educação continuada deve ser uma ferramenta para promover o desenvolvimento das pessoas e assegurar a qualidade do atendimento aos clientes, devendo também ser voltada para a realidade institucional e necessidades do pessoal, visto que foi citado pelos enfermeiros que a proximidade e o contato diário com os técnicos de enfermagem, o interesse pessoal e o da equipe são facilitadores das ações educativas, pois nesse contexto o enfermeiro pode atuar diretamente sobre as necessidades do trabalhador no momento em que este executa suas atividades, percebendo o real interesse da equipe diante das situações cotidianas.

Segundo Bezerra ${ }^{4}$, os especialistas de recursos humanos têm se preocupado, particularmente, com o desenvolvimento de aspectos comportamentais que envolvem as relações de trabalho, tais como: formação, necessidade de treinamento das pessoas, formas de satisfazê-las e fatores motivacionais; visto que a globalização dos mercados mostra que a competitividade está cada vez mais presente em qualquer tipo de empresa, tanto industrializadas como de prestação de serviços, no Brasil e no mundo, sendo as pessoas o recurso mais valioso neste cenário.

Para Chiavenato $^{19}$, no ambiente competitivo, a única vantagem real são as pessoas e só sobreviverão as empresas que considerarem o trabalho humano, não apenas físico, mas o desenvolvimento global. Assim, cada vez mais o conhecimento constitui a força propulsora dos indivíduos nas organizações e na sociedade.

É importante ressaltar que o desenvolvimento das pessoas na área da enfermagem é de responsabilidade do enfermeiro de educação continuada e que o mesmo deve ser o facilitador desse processo, porém, é necessário que as ações educativas propostas sejam compartilhadas entre os enfermeiros e gerentes de serviços, a fim de promover a integração com todos. Também é desejável que o enfermeiro desse Serviço tenha a formação compatível com a de um educador, devendo buscar continuamente o autodesenvolvimento, sendo capaz de influenciar as pessoas na busca do conhecimento e compartilhar seu trabalho com todos os envolvidos na assistência de enfermagem nas instituições de saúde.

\section{Referências}

1. Oguisso T. A educação continuada como fator de mudanças: visão mundial. Nursing 2000;3(20):22-9.

2. Brasil. Ministério da Saúde. Secretaria de Modernização Administrativa e Recursos Humanos. A educação continuada de enfermeiros do SUS. Brasília: Centro de Documentação; 1990.

3. Brasil. Ministério da Saúde. Secretaria de Gestão do Trabalho e da Educação na Saúde. Departamento de Gestão da Educação na Saúde. Política de educação e desenvolvimento para o SUS: caminhos para a educação permanente em saúde: pólos de educação permanente em saúde. Brasília: Ministério da Saúde, 2004. 68 p. (Série C. Projetos, Programas e Relatórios).

4. Bezerra ALQ. 0 contexto da educação continuada em enfermagem na visão dos gerentes de enfermagem e dos enfermeiros de educação continuada. 0 Mundo da Saúde 2000;24(5):352-6.

5. Salum NC, Prado ML. Educação continuada no trabalho: uma perspectiva de transformação da prática e valorização do trabalhador(a) de enfermagem. Texto \& Contexto Enfer 2000;9(2):298-311.

6. Freire P. Pedagogia do oprimido. $12^{\mathrm{a}}$ ed. Rio de Janeiro: Paz e Terra; 1983.

7. Bezerra ALO. Treinamento do enfermeiro recém-admitido: viso do treinador e do treinando. Dissertação (Mestrado). Escola de Enfermagem da Universidade de São Paulo. São Paulo: 1995.

8. Kurcgant P. Educação continuada: caminho para a qualidade. Rev Paul Enferm 1997;16(1/3):72-82.

9. Dalvim RMB, Torres GV, Santos SR. Educação continuada em enfermagem: conhecimentos, atividades e barreiras encontradas em uma maternidade escola. Rev Lat-Americana Enfer 1999;7(5):43-49.
10. Cadete MMM, Villa EA. A concretude da atividade educativa do enfermeiro. 0 Mundo da Saúde 2000;24(5):381-6.

11. Garrido MCF. Cotidiano da educação continuada em enfermagem: valorização do cuidar. 0 Mundo da Saúde 2000;24(5):373-9.

12. Freire P. Pedagogia da autonomia: saberes necessários à prática educativa. ga ed. Rios de Janeiro: Paz e Terra; 1998.

13. Boff L. Saber cuidar: ética do humano - compaixão pela terra. $7^{\mathrm{a}}$ ed. Petrópolis: Vozes; 1999.

14. Gonçalves VLM. Reconstruindo o processo de avaliação de desempenho da equipe de enfermagem do Hospital Universitário da USP. Tese (Doutorado). Escola de Enfermagem da Universidade de São Paulo. São Paulo; 2003.

15. Koizumi MS, Kimura M, Miyadahira AMK, Cruz DALM, Padilha KG, Souza RMC, et al. Educação continuada da equipe de enfermagem nas UTI's do município de São Paulo. Rev Lat-Americana Enfer 1998;6(3):33-41.

16. Gil AC. Como elaborar projetos de pesquisa. $3^{\mathrm{a}}$ ed. São Paulo: Atlas; 1995

17. Conselho Nacional de Saúde. Resolução n. 196, de 10 de outubro de 1996 Diretrizes e normas regulamentadoras de pesquisa envolvendo seres humanos. Bioética 1996;4(2):15-25

18. Santos KMAB, Silva MJP. Percepção dos profissionais de saúde sobre a comunicação com os familiares de pacientes em UTI's. Rev Bras Enfer 2006;59(1):61-6.

19. Chiavenato I. Gestão de pessoas: o novo papel dos recursos humanos nas organizações. Rio de Janeiro: Campos; 1999 